\title{
Capítulo 2
}

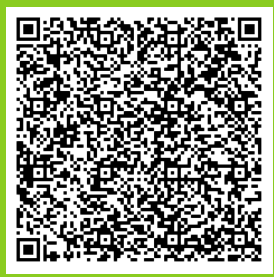




\title{
Políticas Públicas y las Tecnologías de Información y Comunicación en las instituciones educativas de Sincelejo, Sucre, Colombia
}

\author{
Marilú Tibisay Acurero Luzardo ${ }^{1}$ \\ Lucimio Jiménez Paternina² \\ Luis Fernando Jiménez Quintero ${ }^{3}$
}

\section{Resumen}

La Comisión Económica para América Latina y el Caribe-CEPAL, incluye diversos programas para la integración de las Tecnologías de Información y Comunicación - TIC en la educación de los países de América Latina, considerando aspectos que deben ser formulados en las políticas públicas educativas, tal es el caso de la infraestructura técnica, capacidades y conocimientos, contenidos y aplicaciones, regulación e incentivos y lineamientos estratégicos dirigidos, especialmente a la cooperación regional. Por tanto, este estudio analiza las políticas públicas relacionadas con las TIC en las Instituciones Educativas de Sincelejo, Sucre. El estudio es descriptivo de campo no experimental, que busca establecer características fundamentales de conjuntos homogéneos de fenómenos, utilizando criterios sistemáticos para destacar los elementos esenciales de su naturaleza. Finalmente, se concluye con una escasa integración con

\footnotetext{
$1 \quad$ Posdoctora en Políticas Públicas y Paz Social. Doctora en Ciencias Gerenciales. Magister Sienciarum en Gerencia Financiera. Ingeniera de Sistemas. Docente Investigadora de la Corporación Universitaria del Caribe - CECAR, Colombia. Categorizada por Colciencias nivel Asociado. Grupo de Investigación: Estudios Socioeconómicos, Administrativos y Contables. Email: marilu.acurerol@cecar.edu.co; mariluacurero@hotmail.com. Telf. +573147816883. Código ORCID: https://orcid.org/0000-0003-3175-6620

$2 \quad$ Magister en Gestión de Organizaciones. Especialista en Finanzas. Especialista en Revisoría Fiscal. Contador Púbico. Docente Investigador de la Corporación Universitaria del Caribe - CECAR, Colombia. Lucimio.jimenez@cecar.edu.co. Código ORCID: https:// orcid.org/0000-0002-2277-028X.

3 Estudiante de la facultad de Ciencias Económicas Administrativas y Contables Email. Luis.jimenezq@cecar.edu.co
} 
las TIC y un uso no apropiado en la dinámica educativa de Primaria y Secundaria, así como en las Instituciones Educativas oficiales de Sincelejo, producto del desconocimiento y/o la desvalorización de las potencialidades de las TIC.

Palabras clave: Políticas Públicas, Políticas Públicas Educativas y Tecnologías de Información y Comunicación.

\section{Public Policies and Information and Communication Technologies in the educational institutions of Sincelejo, Sucre, Colombia}

\section{Abstract}

The Economic Commission for Latin America and the Caribbean ECLAC, includes various programs for the integration of Information and Communication Technologies - ICT in education in Latin American countries, considering aspects that should be formulated in educational public policies, such as This is the case of technical infrastructure, capacities and knowledge, contents and applications, regulation and incentives and strategic guidelines aimed, especially at regional cooperation. Therefore, this study analyzes the public policies related to ICT in the Educational Institutions of Sincelejo, Sucre. The study is descriptive of a non-experimental field, which seeks to establish fundamental characteristics of homogeneous sets of phenomena, using systematic criteria to highlight the essential elements of their nature. Finally, it concludes with a scarce integration with ICT and an inappropriate use in the educational dynamics of Primary and Secondary, as well as in the official Educational Institutions of Sincelejo, a product of ignorance and/or the devaluation of the potentialities of ICT.

Keywords: Public Policies, Educational Public Policies and Information and Communication Technologies 


\section{Introducción}

La Sociedad de la Información demanda a los Estados, desafíos que les permita conseguir una asimilación adecuada de las Tecnologías de Información y Comunicación - TIC, en la economía, cultura, ciencia y educación, buscando alternativas que consientan incorporar a las TIC a la vida diaria de sus habitantes a través del diseño, realización y valoración de políticas relacionadas con las TIC. Bajo esta perspectiva, las administraciones municipales de Colombia proponen efectivos planes de acción que evolucionan y se desarrollan al mismo ritmo que el resto del mundo.

A pesar de ello, el Departamento de Sucre no se ha orientado a planes efectivos de desarrollo que permitan su expansión y crecimiento, siendo uno de los departamentos con mayor índice de pobreza. Para el año del 2010 reflejó un 63,7\%; en el año 2012 presentó una disminución a un 51,5\%; mostrando un importante descenso de 7,3 puntos. Adicionalmente, para el año 2012, la incidencia de pobreza extrema se mostró en un 5,6\% de la población, cifra, que refleja una reducción porcentual pequeña, pero significativa, ya que para el año del 2010 el 6,4\% se encontraba en pobreza extrema. Paralelo a esto, el desempleo obtuvo una tasa del 10,5\% solo en el Municipio de Sincelejo; que, para el tercer trimestre de 2013, llegó a marcar un 9,9\% (DANE, 2013).

Ahora bien, siendo Sincelejo la capital de uno de los departamentos más pobres de la región y del país, evidencia una situación compleja, toda vez que el grado de analfabetismo constituye uno de los indicadores de desarrollo y calidad de vida de un país, debido a la baja probabilidad que esta población se vincule de marera eficiente al mercado laboral, situación que se refleja en el sistema educativo de la región.

Actualmente, las cifras siguen siendo similares a años anteriores, se debe mejorar la conectividad para facilitar a los ciudadanos el acceso a la información, cifras del DANE y del MinTIC (2018) reflejan que existe una gran diferencia entre los diferentes estratos de la población, para el estrato 1, solo el $20 \%$ de la población tiene acceso al internet a través de banda ancha fija y para el estrato 6 llega al 99.8\%. Ahora bien, según La Unión Internacional de Telecomunicaciones (2017), si se compara a Colombia 
con los países de la región ocupa el lugar 18 entre 26 medidos, por debajo de Ecuador, Jamaica, Perú y Bolivia, cifras estas que no son buenas para el desarrollo.

En consideración a lo expuesto, la Comisión Económica para América Latina y el Caribe (CEPAL, 2013) incluye diversos programas de integración de las tecnologías digitales frente a la educación inclusiva de los países de América Latina, tales como Argentina, Brasil, Chile, Colombia, Costa Rica, México, Panamá y Uruguay; considerandos aspectos que deben ser formulados en las Políticas Públicas educativas, tal es el caso de la infraestructura técnica, capacidades y conocimientos, contenidos y aplicaciones, regulación e incentivos y lineamientos estratégicos dirigidos, especialmente a la cooperación regional.

La aparición de las Políticas Públicas, en cuanto al uso y aplicación de las TIC en la educación en Colombia, se sustenta en la Constitución Política de Colombia (1991), la cual presenta en el Artículo 67 a la Educación como "un derecho de la persona y un servicio público que tiene una función social; con ella se busca el acceso al conocimiento, a la ciencia, a la técnica, y a los demás bienes y valores de la cultura".

Por tanto, el acceso a las TIC en la población estudiantil del Municipio de Sincelejo debe ser un derecho y una prioridad en la agenda de las Políticas Públicas, sobre todo para la población de los estratos bajos, que sería el principal y directamente afectado ante la discriminación al no acceso a las nuevas tendencias educativa, para esto se necesita determinar cuál la realidad actual frente a este problema.

Esta problemática puede ser analizada desde dos dimensiones distintas, por un lado, lo relativo a la brecha digital, la discusión de las TIC como parte del proceso de enseñanza y aprendizaje y por otro lado la discriminación y las posibilidades de inclusión o exclusión social.

En términos concretos, las administraciones locales juegan un papel decisivo en la generación de espacios, que permitan potencializar el sector educativo, con una mayor cobertura y calidad educativa, tomando en consideración las Tecnologías de Información y Comunicación. 


\section{Las Políticas Públicas y las Tecnologías de Información y Comunicación}

El término política se entiende como la norma o reglamentación trazada para alcanzar un propósito determinado. Cuando estas normas se realizan en la gerencia privada, se le llama política empresarial, política organizacional, o política institucional, pero si se conciben en un organismo gubernamental, entonces, serían Políticas Públicas. Salazar (1995), apunta que las Políticas Públicas conforman un conjunto de respuestas por parte del Estado, a las diversas situaciones enmarcadas en problemas sociales. De igual manera, Meny y Thoenig (1992, p. 30) citado por Muñoz y Nuñez (2008), las califican como el producto de las diversas actividades realizadas por una autoridad investida de poder público o poder legítimo de gobierno, presentadas como acciones programadas para un sector de la sociedad o comunidad en un determinado lugar asociado a la comunidad o espacio geográfico.

Aguilar (1992), señala que las políticas públicas se pueden idealizar desde los enfoques descriptivo y teórico; son concebidas desde lo descriptivo se exponen los dictámenes de autoridad dados en una organización política (acuerdos, leyes, órdenes), es por eso que, aunque se generen estrategias y elementos para corregir los problemas que aquejan a una porción de la ciudadanía o a toda esta en general, siempre será el Estado quien fija y decide políticamente. Este enfoque, sostiene que las Políticas Públicas se utilizan a partir de las relaciones que se originan entre el Estado y la sociedad, donde se establecen las relaciones generadas entre los actores sociales y políticos, así como por sus decisiones

La CEPAL plantea una serie de aspectos que deben considerarse en las Políticas Públicas de los países. Dichas políticas deben definir el contexto de este nuevo modelo tecnológico, que propone abordar multidimensionalmente las Políticas Públicas encaminadas a promover las sociedades informacionales, las cuales se encuentran en cinco grupos de variables para la creación de políticas en esta especialidad (CEPAL, 2007).

A continuación, se describen las variables a considerar en la revisión de las Políticas Públicas aplicadas en las instituciones educativas: 


\section{a. Infraestructura técnica}

Según Muñoz y Nuñez (2008), la infraestructura técnica está referida al espacio físico de las personas que utilizan las TIC. La forma de acceso es a través del medio físico, el económico y socio-cultural. El físico se realiza mediante los centros urbanos y las áreas rurales en la región. El económico, es entendido como la disponibilidad de recursos financieros para que personas de distintos niveles de ingreso puedan conectarse, cubriendo el precio de la conexión que incluye los costos de telecomunicaciones, de acceso a Internet y del equipo terminal TIC, tal como computadoras personales, celulares, entre otros. Finalmente, el socio cultural, considerado como una dimensión de la brecha digital que se relaciona con la educación, el origen étnico, el género y la edad, factores que influyen en los patrones de comunicación (p.90).

Asimismo, se identifican tres atributos elementales que facilitan abordar las TIC: como primer aspecto se tiene la propagación de la tecnología móvil la cual por medio del abaratamiento a nivel mundial su masificación ha sido inevitable; el segundo punto es el acceso social para el que los países han implementado tres maneras de permitir el acceso popular a Internet estas son: oferta directa por parte de los gobiernos, subsidiar una mínima parte a través de fondos de acceso global, y los fondos de inversión, permiten que las empresas a nivel local compitan entre sí se incrementen los centros de acceso público. El tercer punto está dedicado a las nuevas tecnologías basadas en protocolos como el Wireless Application Protocol (WAP) el cual permite el acceso a Internet a las poblaciones vulnerables con problemas de alfabetización. Por su parte la Open Mobile Alliance - OMA trabaja en la implementación de tecnologías como lo es 4G y 5G (telefonía de cuarta y quinta generación) la cual, según Guevara (2019) por el bajo consumo energético y su fácil implementación, hacen que esta tecnología sea bastante beneficiosa porque permitiría que muchas más personas tuvieran acceso a esta tecnología y la televisión digital interactiva a la que la gran mayoría de los habitantes tiene acceso, por lo que su potencial es prometedor

De igual forma, la red $5 \mathrm{G}$ propone un mayor ancho de banda, más espaciamiento y velocidad, generando un menor tiempo de respuesta, la interferencia disminuye casi en su totalidad para una transmisión adecuada, 
las formas de onda y su enfoque aplicado a las diversas técnicas, con agilidad de la transmisión en tiempo real, generando mejoras sobre la transmisión convencional (Guevara, 2019, p.64).

\section{b. Potenciales y Técnicas Codificadas y Transmitidas de forma estandarizada}

En términos de potenciales y técnicas codificadas se hace necesario aclarar varios puntos para entender cómo se puede emplear la infraestructura tecnológica instalada. Lo competente a la capacidad de admisión de las TIC hay que tener claro que Las PYMES producen entre un $40 \%$ y $50 \%$ del producto interno bruto (PIB) en Latinoamérica, y que, para alcanzar las metas en el incremento productivo y el decrecimiento del desempleo regional, obedecerá en gran medida del potencial de sostenimiento y crecimiento en el mercado. Es de esperar, entonces, que las PYMES de un Estado prosperen e incrementen sus ganancias si acogen en masa las TIC dentro de sus procesos administrativos, productivos y comerciales.

Por lo tanto, es trascendental crear conciencia dentro de los que hacen empresa, sobre la aplicación de las TIC; por lo que nace una propuesta basada en tres (3) rangos afines con el grado de aceptación de las TIC a nivel empresarial: Sin red, con intranet y con extranet por lo que es relevante establecer las ventajas de adoptar un cambio en tecnología, su coste y los cambios requeridos dentro de cada organización. Por lo tanto, es responsabilidad del Gobierno estatal diseñar e implementar políticas públicas que proporcionen el desarrollo tecnológico, dependiendo de la capacidad de cada empresa, suministrando los recursos y las herramientas institucionales requeridas, todo esto producto de que el Internet es Dado que el acceso a Internet es aprovisionado por compañías externas, por lo tanto se considera que su acceso no genera un cambio reconocido dentro de las PYMES y su organización (Muñoz \& Núñez, 2008).

En cuanto a la educación que permite utilizar las TIC, existen los grados de alfabetización informática básica, media y avanzada. Al usar las expresiones como educación básica, media o avanzada u otros obtenidos de la formación escolar, no se debe confundir la alfabetización y formación informática con la educación escolar. Dichos términos en el medio de la formación informática tienen un sentido propio y las entidades y recursos 
pedagógicos que requieren no son esencialmente los mismos que los de la educación escolar. Esto no imposibilita que la educación digital utilice las instituciones educativas, escolares o no, para conseguir sus metas.

El nivel básico contiene algunos rasgos como lo son: desarrollo pedagógico en relación con personas que dominan la materia (docentes); el software es intuitivo; el adiestramiento es especialmente empírico; la alfabetización informática es el producto de la operación de un computador y no se precisa de estímulos para jóvenes y adultos. El nivel intermedio demanda recursos más avanzados, como docentes mejor capacitados que compartan conocimiento ordenado, alumnos autodidactas, procesos persistentes y frecuentes y el estudiante contribuye con tiempo de amaestramiento.

El nivel avanzado contiene fundamentos más complicados que el nivel intermedio, la educación es caracterizada en prácticas determinadas lo cual conlleva delimitar áreas de enseñanza que a su vez comprende el diseño de estrategias que requieren personal competente. La inversión en una mejor infraestructura y la incentivación adecuada deben ser políticas públicas que el Estado requiere implementar, en busca de la transformación a la era digital. Creando ciudadanos capaces de enfrentar de competentemente a la nueva sociedad de información (Muñoz \& Núñez, 2008).

Por otro lado, se expone el planteamiento de si los grupos objetivo de las políticas de alfabetización informática deben adaptarse a la demanda, si requieren la creación de una oferta autónoma o si es necesario educar a toda la ciudadanía sin importar las condiciones de mercado.

El sistema educativo ha evolucionado en su amplitud o cobertura lo cual invita a que se logre la alfabetización informática en las diversas instituciones. Se hace imperante establecer programas de formación dirigidos a los diferentes niveles educativos, tales como: Primaria, básica, Secundaria y hasta niveles de educación superior, tomando en cuenta las diferencias que puedan encontrarse.

\section{c. Compendios y Aplicativos Públicos}

El nuevo modelo consume procesa, almacena y distribuye datos a niveles que ninguna empresa o ente privado alcanzaría por lo que su impacto en el aparato estatal es de repercusiones gigantescas. Por tanto, los 
legisladores de una sociedad democrática representativa deben enfocar su mayor esfuerzo en obtener flujos constantes de información y metodologías comunicativas, directas con la ciudadanía en general.

Países de América Latina como Argentina, Brasil, Colombia y Chile, según cifras de la CEPAL (2005), han aumentado en consideración la dotación de ordenadores y el acceso a la gran red (internet), el problema es que no utilizan correctamente esta infraestructura, el desarrollo de software, la elaboración de aplicativos educativos, no son los más apropiados, incidiendo directamente en la evolución y gestión del conocimiento en todas las áreas. A diferencia de otras regiones del mundo, los sistemas educativos en Latinoamérica están basados en un pequeño número de idiomas, lo que facilita el intercambio de contenido educativo y de aplicaciones científicas. Esta red intenta hacer el intercambio de contenidos productos del trabajo de diferentes Naciones y sus recursos culturales y educativos, por lo que muchos de estos contenidos sufren un proceso adaptativo dependiendo de cada país (Muñoz \& Nuñez, 2008).

\section{d. Herramientas Incentivadoras y Reguladas}

Entre los instrumentos utilizados para impulsar el uso de TIC se encuentran cuatro propiedades:

- El aporte de los recursos económicos indispensables para la ejecución de los proyectos de desarrollo en la infraestructura TIC.

- El desarrollo de potenciales y acceso a la información.

- La digitalización de los procedimientos.

- La prestación de servicios públicos mediante redes digitales. Se consideran los procedimientos y requerimientos necesarios para establecer los mecanismos de coordinación dentro del marco institucional de una estrategia nacional.

No obstante, la CEPAL (2005) concibió que las oportunidades de financiación pueden aumentar si se consideran los siguientes fundamentos:

- Inversiones privadas que funcionan al límite del arbitraje público encaminado concretamente a las TIC. 
- Inversiones privadas promovidas por estrategias públicas que no involucran recursos estatales.

- Inversiones públicas que incluyen acciones directas del gobierno subsidiando; y con apoyo financiero internacional

Ahora bien, Muñoz \& Núñez (2008), manifiestan de la necesidad de invertir en cuanto a la adopción de tecnologías, para lo cual se ha dispuesto de algunas regulaciones en las tarifas para el uso de las TIC en los diferentes sectores de la población; de igual forma se motiva a las personas en la adquisición de las TIC mediante los incentivos tributarios, reducción de aranceles o mecanismos de contribución obligatoria, como los fondos de universalización de acceso financiados del ingreso de los operadores de las telecomunicaciones y fondos tecnológicos sectoriales, financiados vía presupuestaria o por contribuciones privadas (p.98).

Es indispensable resaltar que estas herramientas reguladoras, de financiación y marco jurídico están orientadas hacia la implementación de una sociedad digital con el uso de TIC por lo que se deben crear instrumentos de medida y seguimiento que proporcionen la visualización y la evolución en los trabajos que se están ejecutando entre los que se hallan: el Observatorio para la Sociedad de la Información en Latinoamérica y el Caribe (OSILAC), el cual está fundamentado en una disposición de la Conferencia Estadística de las Américas (CEA), cuyas metas principales son la de agrupar datos, indicadores, procesos e información cualitativa derivada de toda la región; regularizar y equilibrar a diversos niveles territoriales indicadores TIC y optimizar la calidad de la información TIC.

\section{Pública \\ e. Reglamentación Estratégica y sus Alcances en la Política}

La conexión entre Estado y las TIC conlleva el fomento de la evolución de la sociedad hacia la usanza de la información enfocándose en el desarrollo económico con entereza y la conversión digital del mismo Estado en la búsqueda infinita de la eficiencia. Lo que generaría el espacio a Políticas Públicas que se fortalezcan con una participación regional más intensiva. El planteamiento de políticas públicas que generan más oportunidades de acceso a las TIC es refrenado debido a la evolución de las mismas, escondidas 
por los conflictos de gobernabilidad, la poca participación de la sociedad civil y de las empresas privadas y lo complicado de las propiedades de la tecnología.

\section{f. Restricciones Regionales y la Política Pública para el Acceso a las TIC}

Se consideran los siguientes aspectos:

1. El crecimiento y evolución de la telefonía móvil

2. Conectividad a través de las redes inalámbricas - WiFi

3. Redes de informática con acceso a Internet con ancho de banda en sistemas WIFI en puntos de acceso público

4. Explorar nuevas opciones, como la Televisión Digital Terrestre

Las herramientas institucionales y los emprendimientos de adiestramiento deben ser aprovechados y consolidados con coaliciones estratégicas nacionales e internacionales, con la empresa privada y la sociedad civil, lo que podría incentivar los esfuerzos para educar a la sociedad para y en la era digital. Los esfuerzos de adiestramiento informático se deben concentrar en personas clave, como empleados públicos, docentes, que, por su concurrente contacto con la comunidad, pueden llevar las bondades de las TIC a muchos sectores de población (Muñoz \& Nuñez, 2008).

Con respecto a la tendencia y el uso de prototipos de software la práctica indica que la política pública es de alta importancia en materia investigativa, y en el estímulo al desarrollo de modelos abiertos y convergentes con los que existen a nivel internacional es primordial, desde luego es necesario precisar el uso de software con código abierto (Open Source). En relación con la implementación de sociedades informacionales es necesario tener Políticas Públicas que amplíen el acceso a las TIC, fortificar la capacidad, la eficacia y transparencia del Estado, los cuales tienen repercusiones regulatorias muy amplias, siendo este uno de los más grandes retos, teniendo en cuenta las características del tipo de conectividad precisada en la región (CEPAL, 2005, p. 44).

El impacto de las Tecnologías de Información y Comunicación (TIC) en la ciencia, la economía y la cultura, y por ende en la educación, como parte de la llamada Sociedad de la Información, exige a las naciones nuevos 
retos para lograr una apropiación adecuada y buscar alternativas que permitan incorporarlas a la vida cotidiana de sus ciudadanos a través de la formulación, ejecución y evaluación de políticas relacionadas con las TIC. Bajo esta perspectiva las administraciones municipales de Colombia, deben realizar planes efectivos que permitan evolucionar y desarrollarse al mismo ritmo que el resto del mundo.

Caracterizar las diferentes políticas en materia educativa, puede ser el punto de partida para conocer la efectividad de esta, teniendo en cuenta además la necesidad de optimizar los recursos económicos; en el Municipio de Sincelejo, no se tiene claramente definida la efectividad de las políticas en inversión en las TIC, por lo cual esta investigación, mostrará una "radiografía" general de como se ha venido invirtiendo en calidad educativa, como estamos en el presente, para así tener bases sólidas a la hora de toma de decisiones para el futuro.

El propósito de esta investigación es dar a conocer las principales características de las Políticas Públicas desarrolladas en el Municipio de Sincelejo, Sucre en relación con las Tecnologías de la información y la comunicación con el objetivo de mejorar la calidad educativa; para tal efecto se desarrolló una investigación, descriptiva, conceptualmente se fundamentó en un conjunto de variables propuestas por la CEPAL que serán utilizadas en este trabajo para caracterizar las políticas públicas. Dichas características delimitan un contexto complejo del actual modelo tecnológico, por lo que se plantea abordar multidimensionalmente las políticas públicas encaminadas a promover las sociedades de la información en cinco agrupaciones de variables para el desarrollo de políticas en este ámbito (CEPAL, 2013).

\section{Metodología del estudio}

Este estudio es descriptivo de campo no experimental, que busca establecer características fundamentales de conjuntos homogéneos de fenómenos, utilizando criterios sistemáticos para destacar los elementos esenciales de su naturaleza (Fernández, Baptista \& Hernández; 2014). En este caso se realiza un análisis sobre la aplicación de las políticas públicas educativas relacionadas con las TIC en el Municipio de Sincelejo. Asimismo, se señala que mediante este tipo de investigación se obtiene 
un mayor conocimiento del fenómeno objeto de estudio, se describen las características más importantes del mismo en lo que respecta a su aparición, frecuencia y desarrollo.

En consideración a las políticas públicas educativas, se construye una matriz de caracterización, tomando en cuenta las variables sociedad de la información, definidas en el marco de referencia. Para los datos subsiguientes se escogió se realiza un análisis documental análisis documental siguiendo la metodología aplicada por Barboza, Barboza y Rodríguez (2013).

La revisión documental se formalizó examinando archivos físicos y electrónicos del Departamento Nacional de Planeación, Ministerio de Ciencia Tecnología y Comunicación, Ministerio de Educación Nacional, Secretaria de Educación de Sincelejo, dependencias que tienen oficio y responsabilidad de ordenar, ejecutar y hacer seguimiento de políticas públicas educativas relacionadas por las TIC.

\section{Análisis de resultados}

La caracterización de las Políticas Públicas formuladas sobre las bases de las TIC se apoyó en un análisis documental donde se realiza una revisión externa e interna sobre los aspectos resaltantes de la política inmersos en los diversos documentos. Para el Primer nivel se detallan los documentos de la Política Pública y se registran los siguientes datos: título, responsable de la elaboración, fecha de promulgación o divulgación y en el segundo nivel se obtienen datos sobre el contenido documental como objetivos, resumen, temas generales tratados, políticas públicas formuladas sobre las TIC, educación y observaciones que puntualizaron el contenido temático y la visión elaborada en el estudio. De igual manera, en este punto se añaden al formato elementos concernientes con las características de políticas públicas de las que hablan Meny y Thoenig (1992).

Ahora bien, en las tablas No. 1, 2, 3 y 4 se describen los aspectos relacionados a las características de las Políticas Públicas los cuales son analizados en las diversas Instituciones Educativas de la ciudad de Sincelejo. 


\section{Tabla 1}

\section{Infraestructura Técnica}

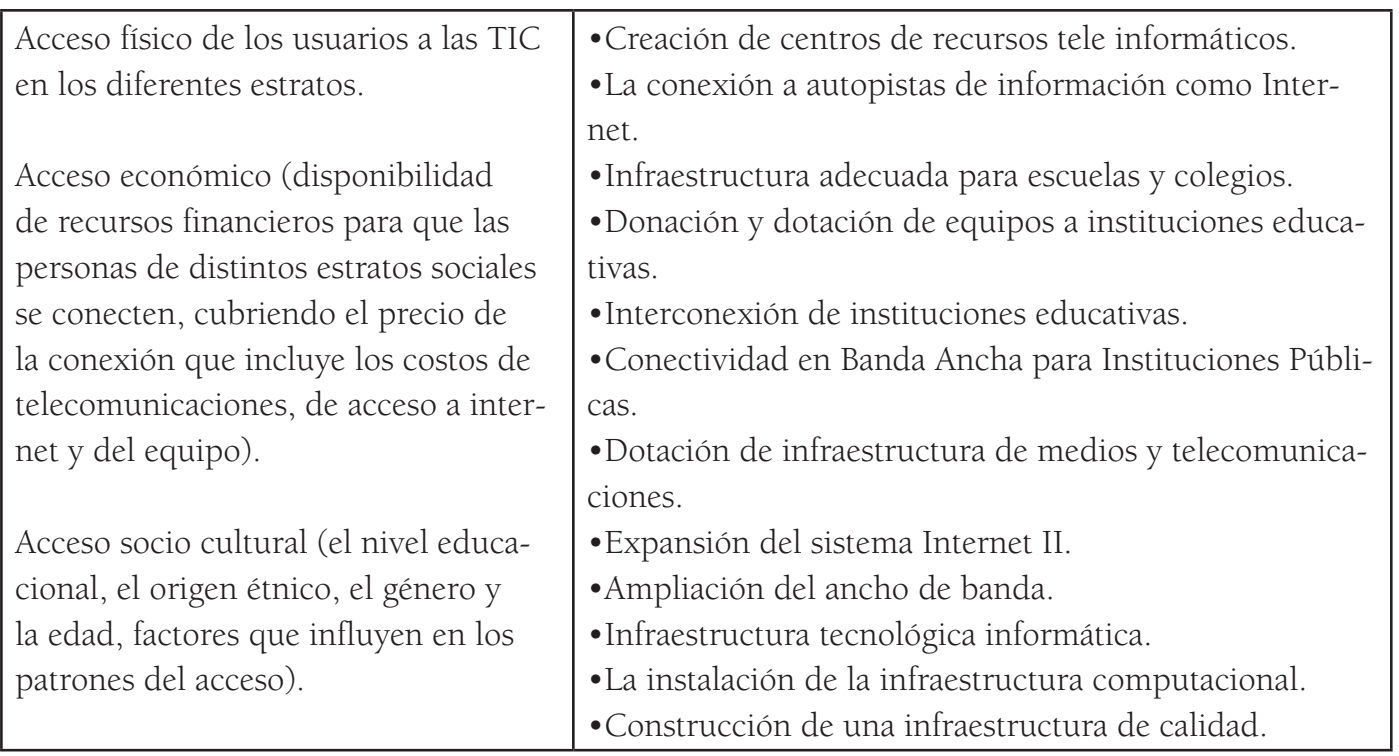

Fuente: Elaboración propia.

Tabla 2

Capacidades y conocimientos codificados y transmitidos en estándares

\begin{tabular}{|c|c|}
\hline $\begin{array}{l}\text { Dilucidar diferentes } \\
\text { elementos para } \\
\text { comprender cómo } \\
\text { se puede usar la } \\
\text { infraestructura tec- } \\
\text { nológica instalada. }\end{array}$ & $\begin{array}{l}\text { - Desarrollar el programa de Integración a las redes y programas } \\
\text { internacionales de investigación y desarrollo tecnológico. } \\
\text { - El uso de los medios masivos de comunicación, especialmente la } \\
\text { televisión, la informática y las redes telemáticas. } \\
\text { - Difusión masiva de los programas educativos a través de la radio, } \\
\text { los canales públicos locales y regionales o la televisión por cable. } \\
\text { - Incremento del uso de los equipos obtenidos por diversos pro- } \\
\text { gramas como herramienta de enseñanza en colegios y escuelas } \\
\text { públicas. } \\
\text {-Propuesta de un plan de capacitación docente para el uso de las } \\
\text { tecnologías de la información y comunicación. } \\
\text { - Fomento de redes escolares nacionales. } \\
\text { - Adecuados esquemas pedagógicos y requerimientos educativos } \\
\text { para el uso de TIC } \\
\text { - Creación y fortalecimiento de proyectos dirigidos al aprendizaje } \\
\text { colaborativo con ayuda de las TIC. }\end{array}$ \\
\hline
\end{tabular}

Fuente: Elaboración propia. 
Tabla 3

\section{Contenidos y aplicaciones públicas}

El nuevo paradigma puede tener un profundo impacto en el Estado pues éste, para poder funcionar, consume, almacena, procesa y distribuye información en escalas que ninguna empresa $\mathrm{o}$ entidad privada puede alcanzar. El trabajo del poder legislativo de una democracia representativa está basado casi totalmente en flujos de información y procesos de comunicación entre los representantes del pueblo o con los ciudadanos.
- Renovación del currículo previo a la revisión de los programas académicos de las áreas obligatorias y fundamentales del conocimiento y de la formación.

- Fomento a la producción y el uso de contenidos educativos digitales.

- Desarrollo de propuestas pedagógicas que estimulen en niños y jóvenes el espíritu científico, las prácticas de investigación y la creatividad para la innovación tecnológica.

- Diversificación de la oferta de contenidos y servicios a través de portales, bancos de objetos de aprendiz aje y bodegas de materiales educativos digitales ampliando el alcance de la cooperación entre países.

- Fomento de la presencia en línea de los participantes del sistema educativo

- Uso de nuevas tecnologías, radio y televisión dentro de los espacios educativos

Fuente: Elaboración propia. 


\section{Tabla 4}

\section{Lineamientos estratégicos y sus implicaciones de política pública}

Entre las herramientas para promover

el uso de las TIC se encuentran cuatro aspectos:

1) El refinanciamiento de los recursos financieros

necesarios para la implementación de los proyectos de construcción de la infraestructura TIC

2) La creación de capacidades y acceso a los conocimientos.

3) La digitalización de procesos.

4) La prestación de servicios públicos mediante redes

digitales e incluso para asegurar la operación de los mecanismos de coordinación necesarios para establecer el marco institucional de una estrategia nacional para la sociedad de la información.
-Apoyo en los planes de fortalecimiento de infraestructura tecnológica en las instituciones mediante créditos de entes gubernamentales de responsables de manejo recursos financieros.

- Diseño de una línea de crédito para facilitar el acceso a

Computadores personales a la población de bajos ingresos.

- Proporcionar conectividad de banda ancha a redes a los centros educativos públicos y privados de preescolar, básica y media con tarifas subsidiadas de acuerdo con la capacidad económica.

- Negociar con proveedores, computadores de bajo costo

para instituciones educativas, docentes y estudiantes.

Fuente: Elaboración propia.

Revisando además otros documentos sobre planes y proyectos pertinentes en cuanto a las Políticas Públicas y teniendo en cuenta las variables propuestas por la CEPAL se identificaron los siguientes aspectos; donde se muestra el nombre de la acción y el Estado actual. Se tomó en consideración solo las que se encuentran en ejecución, la entidad quien lo ejecuta, la descripción general y la categorización de la CEPAL.

\section{Plan de acción de la Secretaría de Educación y Cultura - 2017, de la Alcaldía de Sincelejo:}

Con relación a la conectividad de las escuelas de Sincelejo, (35) de las Instituciones Educativas Oficiales tienen servicio de internet a través de Tigo UNE, el 69\% (24) se encuentran en la zona urbana y el 31\% (11) en la zona rural. De las 98 sedes, solo (96) están conectadas, representa el 98\%. Es decir, se garantiza conectividad en la zona urbana a todas las sedes donde existía dotación de computadores y sala de informática. 
El 1\% de sedes no atendidas no cuentan con sala de informática o dotación de computadores. Las sedes rurales no principales, se han puesto a disposición del programa puntos digitales por tener la garantía de conectividad en esas sedes distantes y en donde no había salas o equipos de cómputo.

El grave problema que afecta la población estudiantil del Municipio de Sincelejo, es la escasa posibilidad de acceso a la educación Primaria, Secundaria y media vocacional y el inadecuado espacio que existe para desarrollar el apoyo logístico. La alternativa contempla la creación de escuelas y mejoramiento de las existentes, que traducido se convierte en la ejecución de obras preliminares, estructuras en concreto, mejoras en términos generales que conviertan las infraestructuras en espacios dignos para la educación.

En la actualidad Las Instituciones Educativas del Municipio de Sincelejo seleccionadas por el programa COMPUTADORES PARA EDUCAR cuentan con aulas escolares que no se encuentran en las condiciones adecuadas para prestar sus servicios. La alternativa contempla el suministro de piso en cerámica, pintura en vinilo tipo 1, pintura para puertas, suministro e instalación de aire acondicionado, instalación de polo a tierra, y el suministro de elementos necesarios utilizados en aulas de informática.

Debido a la insuficiente capacidad de infraestructura que se tiene en la ciudad de Sincelejo para cubrir la demanda de la población estudiantil y sus instalaciones en mal Estado hace que se agudice esta situación, esto se evidencia cada vez más a medida que transcurren los años, ya que la población crece y es mayor la necesidad de los cupos para los alumnos que luchan por conseguir el ingreso al plantel educativos, es por ello que se hace de vital importancia la adecuación de la sala de informática en el Teatro Municipal de la ciudad de Sincelejo. Se adecuará un aula para una sala de informática con $60 \mathrm{ml}$ de canaleta, 20 salidas de toma corriente, suministro de lámparas, entre otros trabajos.

El objetivo principal del plan es la recuperación del componente académico, como uno de los factores esenciales de la calidad de la educación que reciben los estudiantes matriculados en las distintas Instituciones 
Educativas de carácter oficial del Municipio de Sincelejo, mediante la adquisición de computadores portátiles que permitan mejorar los procesos de formación y actualización de los docentes y estudiantes.

En el Municipio de Sincelejo, la secretaria de Educación Municipal como entidad certificada por el Ministerio de Educación Nacional está en la obligación de mejorar la calidad del servicio educativo que se presta en las distintas instituciones educativas, pero en éstas existe un alto nivel de atraso tecnológico, los equipos de computación que existen, en su mayoría ya cumplieron su vida útil, presentan dificultades e incompatibilidades en los sistemas operativos con respecto a programas y paquetes tecnológicos actualizados.

Se promueve la integración, uso y apropiación de las Tecnologías de la Información y las Comunicaciones, en la dinámica educativa de las Instituciones Educativas Ofíciales del Municipio de Sincelejo.

Además, se observa un atraso significativo en los instrumentos que incluyen incentivos y regulación, y en los lineamientos estratégicos y sus implicaciones de Políticas Públicas. Esto puede tener un efecto directo en el desarrollo de las capacidades de los estudiantes de la Ciudad de Sincelejo, debido a que, no cuentan con el conocimiento necesario para transmitir el cambio de cultura en cuanto al uso de las TIC.

La Secretaria de Educación Municipal, expidió la Resolución Nº946 de 2013, por medio del cual se implementa, desarrolla y sostiene el macro proceso gestión de calidad del servicio educativo en educación preescolar, básica y media, aquí se establecen lineamientos, actividades, recursos, metodologías a utilizar en ellas y se designan funcionarios que realizan el acompañamiento y asistencia técnica en los establecimientos educativos del Municipio de Sincelejo.

El propósito del Ministerio de Educación Nacional y de la Secretaría de Educación Municipal de Sincelejo para el año 2014, es el que todos los establecimientos educativos oficiales y privados, implementen el proceso de Gestión de la Calidad del Servicio Educativo en Educación Preescolar, Básica y Media como una nueva herramienta de gestión; para ello, cada establecimiento educativo debía planificar y llevar a cabo la realización 
de los procesos y bajo condiciones controladas por el equipo del área de Calidad Educativa, que permitan evidenciar el cumplimiento de los acuerdos establecidos y así garantizar la prestación efectiva del servicio.

\section{Relación de las Políticas Públicas asociadas a las TIC en la educación del Municipio Sincelejo, Sucre}

- Las políticas son formuladas con base en lineamientos trazados desde organismos internacionales como la CEPAL, pues documentos de esta organización señalan que la relación entre Estado y las TIC dan lugar a agendas de política que pueden integrarse en un contexto regional y están formuladas para responder a la orientación de la CEPAL, en el sentido de buscar la integración regional de agendas para las sociedades de la información, que puede ser un poderoso instrumento para el crecimiento económico con equidad (CEPAL, 2005, p. 12).

- Las políticas públicas que muestran un mayor nivel de ejecución son las correspondientes a infraestructura técnica, mientras que las relacionadas con las variables de capacidades y conocimientos, al igual que las de contenidos y aplicaciones, se han formulado recientemente y sólo aparecen en el papel de la norma o del documento de planeación.

- Lo afirmado por Brunner (2000) en el sentido de que las TIC están revolucionando la educación en el mundo tiene vigencia plena, ya que las comunicaciones están replanteando el esquema espaciotemporal de los actores de la educación.

- Las TIC representan bondades en el entorno educativo, pero también generan problemas que deben ser resueltos desde las políticas públicas. Hacen referencia a aspectos fundamentales: la equidad en lo que proporciona el acceso a las TIC; contextos para la definición de los currículos, pues surgen tensiones entre los contextos locales y los globales a los que los currículos deben responder. 


\section{Caracterizar las TIC mediante la metodología utilizada por la CEPAL}

Consolidando esta información bajo la metodología de categorización planteada por CEPAL se obtuvo el siguiente resultado:

\section{Tabla 5}

Categorías de la CEPAL

\begin{tabular}{lc}
\hline \multicolumn{1}{c}{ CATEGORÍAS CEPAL } & PORCENTAJES \\
\hline Infraestructura técnica & $67 \%$ \\
Capacidades y conocimientos codificados y transmitidos & $22 \%$ \\
en estándares & \\
Contenidos y aplicaciones publicas & $11 \%$ \\
Instrumentos que incluyen incentivos y regulación & $0 \%$ \\
Lineamientos estratégicos y sus implicaciones de Políticas & $0 \%$ \\
Publicas & \\
\hline
\end{tabular}

Fuente: Elaboración propia.

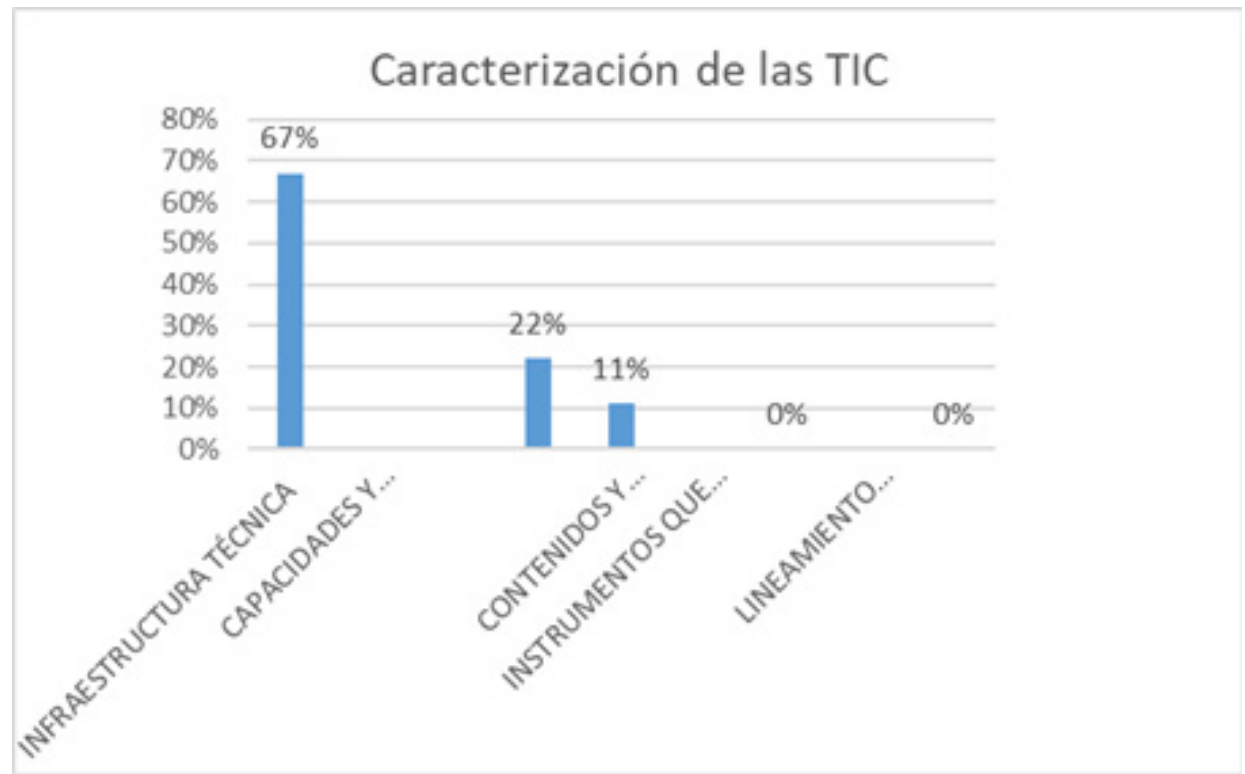

Figura 1. Caracterización de las TIC

Como muestra la figura 1 más de la mitad de los proyectos desarrollados como Políticas Públicas relacionadas con las TIC en la calidad educativa del Municipio de Sincelejo, se basan principalmente en la dotación de infraestructura técnica (al rededor del 67\% aproximadamente), situación que refleja un poco el atraso en la implementación de mecanismos de 
capacitación. El siguiente 22\% son políticas orientadas a las capacidades y conocimientos codificados y transmitidos en estándares; y el 11\% en contenidos y aplicaciones públicas.

\section{Conclusiones}

Se observa una escaza integración, uso y apropiación de las Tecnologías de Información y de Comunicación, en la dinámica educativa de Primaria y Secundaria en las Instituciones Educativas oficiales del Municipio de Sincelejo, departamento de Sucre, producto del desconocimiento y/o desvalorización de las potencialidades de las TIC en el proceso de enseñanza y aprendizaje, la resistencia al cambio en la dinámica educativa por parte de docentes y alumnos y por último el limitado acceso a equipos y recursos informáticos. Todas estas situaciones negativas se ven reflejadas en la poca preparación de los estudiantes de las Instituciones Educativas oficiales para enfrentar una educación globalizada. Lo que trae como consecuencia el bajo desarrollo socioeconómico del Municipio de Sincelejo.

Se requiere del incentivo al uso de las TIC como herramienta pedagógica, para lo cual se necesitan de programas educativos necesarios para la enseñanza. Así como de la ampliación, construcción y/o mantenimiento de la infraestructura apropiada para el uso de las TIC. Es de hacer notar que para facilitar el acceso al conocimiento universal y al uso de las TIC se hace necesario garantizar el acceso a internet en las Instituciones Educativas oficiales.

Los altos niveles de desigualdad en los diferentes estratos sociales de la población inciden en la educación de los jóvenes de las instituciones educativas. En cuanto a los términos económicos se observa una baja inversión de recursos, un inadecuado uso y poco aprovechamiento de las TIC, incidiendo marcadamente al incremento de la brecha digital entre los diferentes estratos de la población. 


\section{Referencias}

Aguilar, L. (1992). La Hechura de las Políticas. Miguel Angel Porrua Grupo Editorial.

Barbosa Chacón, J. W., Barbosa Herrera, J. C. \& Rodríguez Villabona, M. (2013). Revisión y análisis documental para estado del arte: una propuesta metodológica desde el contexto de la sistematización de experiencias educativas. Investigación bibliotecológica, 27(61), 83105. http://www.scielo.org.mx/scielo.php?script=sci_arttext\&pi$\mathrm{d}=$ S0187-358X2013000300005\&lng=es\&tlng=es.

Brunner, J. J. (2000, 23 al 25 de agosto). Globalización y el futuro de la educación: tendencias desafios, estrategias. Seminario sobre Prospectiva de la Educación en la Región de América Latina y el Caribe, Santiago de Chile, Chile. http://www.schwartzman.org.br/simon/delphi/ pdf/brunner.pdf

Comisión Económica para América Latina y el Caribe [CEPAL]. (2005). Políticas públicas para el desarrollo de sociedades de la información en América Latina y del Caribe (LC/W.19). https://www.cepal.org/ es/publicaciones/3577-politicas-publicas-desarrollo-sociedades-informacion-america-latina-caribe

Comisión Económica para América Latina y el Caribe [CEPAL]. (2007). Monitoreo del eLAC2007: avances y Estado actual del desarrollo de las sociedades de la información en América Latina y el Caribe (LC/W.151). https://www.cepal.org/es/publicaciones/3584-monitoreo-elac2007-avances-estado-actual-desarrollo-sociedades-la-informacion

Comisión Económica para América Latina y el Caribe [CEPAL]. (2013). Las tecnologías digitales frente a los desafios de una educación inclusiva en América Latina. Algunos casos de buenas prácticas (LC/L.3545268). https://www.cepal.org/es/publicaciones/21658-tecnologias-digitales-frente-desafios-educacion-inclusiva-america-latina-algunos

Constitución Política de Colombia. (1991). Gaceta Constitucional n. ${ }^{\circ} 116$. http://bit.ly/2NA2BRg

Departamento Administrativo Nacional de Estadística [DANE] . (2013). SUCRE: POBREZA MONETARIA 2013. https://www.dane.gov.co/files/ 
investigaciones/condiciones_vida/pobreza/Sucre_Pobreza_2013. pdf

Fernández Collado, C., Baptista Lucio, P. \& Hernández Sampieri, R. (2014). Metodología de la Investigación. Editorial McGraw Hill.

Guevara Córdoba, F. A. (2019). Comparativo entre la tecnología de redes $4 G$ y $5 G$ y los beneficios de su implementación en Colombia [Tesis Doctoral, Universidad Santiago de Cali]. Repositorio USC. https:// repository.usc.edu.co/handle/20.500.12421/189

Meny, Y. \& Thoenig, J. C. (1992). Las políticas públicas. Editorial Ariel.

Ministerio de Tecnologías de la Información y las Comunicaciones [MinTIC]. (2018). Plan TIC 2018 - 2022. El Futuro Digital es de Todos. https://micrositios.mintic.gov.co/plan_ tic_2018_2022/pdf/plan_tic_2018_2022_20200107.pdf Muñoz Rojas, H. A., \& Nuñez Valero, J. G. (2008). Caracterización de las políticas públicas educativas relacionadas con Tecnologías de la Información y la Comunicación, TIC, en Colombia [Tesis de Maestría, Universidad de La Salle]. Repositorio La Salle. https:// ciencia.lasalle.edu.co/maest_docencia/631/

Salazar, C. (1995). Las políticas públicas. Nueva perspectiva de análisis. Ciencia política: Revista trimestral para América Latina y Espana, (38), 59-79. https://dialnet.unirioja.es/servlet/articulo?codigo $=4415515$

Unión Internacional de Telecomunicaciones. (2017). Measuring the Information Society Report 2017 Volume 1. ITU. https://www.itu.int/en/ ITU-D/Statistics/Documents/publications/misr2017/MISR2017_ Volumel.pdf 